\title{
Authentication of collagen VI antibodies
}

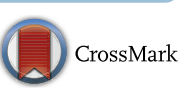

\author{
Jamie Endicott ${ }^{1}$, Paul Holden ${ }^{2}$ and Jamie Fitzgerald ${ }^{1,2^{*}}$
}

\begin{abstract}
Background: Collagen VI is a ubiquitously-expressed macromolecule that forms unique microfibrillar assemblies in the extracellular matrix. Mutations in the COL6A1, COL6A2 and COL6A3 genes result in congenital muscular dystrophy, arguing that collagen is critical for skeletal muscle development and function. Antibodies against collagen $\mathrm{VI}$ are important clinical and diagnostic tools in muscular dystrophy. They are used to confirm genetic findings by detecting abnormalities in the distribution, organization and overall levels of collagen VI in cells and tissues isolated from patients.

Methods: Many antibodies have been raised against tissue-purified collagen VI and individual collagen VI chains, however few have been properly validated for sensitivity and chain specificity. To address this deficiency, we compared the ability of 23 commercially-available antibodies to detect extracellular collagen VI by immunohistochemistry on frozen tissue sections. To determine chain specificity, immunoblot analyses were conducted on cell lysates isolated from cells transfected with cDNAs for each individual chain and cells expressing all three chains together.

Results: Our analyses identified 15 antibodies that recognized tissue collagen VI by immunohistochemistry at varying intensities and 20 that successfully detected collagen VI by immunoblotting. Three antibodies failed to recognize collagen VI by either method under the conditions tested. All chain-specific antibodies that worked by immunoblotting specifically recognized their correct chain, and no other chains.

Conclusions: This series of side-by-side comparisons reveal at least two antibodies specific for each chain that work well for immunohistochemistry on frozen sections. This validation study expands the repertoire of antibodies available for muscular dystrophy studies caused by defects in collagen VI.
\end{abstract}

Keywords: Collagen VI, Muscular dystrophy, Immunohistochemistry

\section{Background}

A major discussion point within the biomedical research community concerns experimental reproducibility. One recent study attempted to replicate the findings from 53 clinical experiments but found that results from just six of these studies could be independently confirmed [1]. While there are many reasons for this, a primary factor is the inadequate validation of antibodies leading to false positive (and negative) findings. Many antibodies in the public domain have been found to be poorly characterized. For example, a 2008 study found that only half of $>6000$ commercially-available antibodies uniquely

\footnotetext{
*Correspondence: jitzge2@hfhs.org

${ }^{1}$ Bone and Joint Center, Department of Orthopedic Surgery, Henry Ford Hospital System, Integrative Biosciences Building, 6135 Woodward Ave, Detroit, Ml 48202, USA

Full list of author information is available at the end of the article
}

recognized their stated targets [2]. The most common issue was poor specificity, where the antibody recognizes structurally-similar proteins in addition to the target protein. As the use of antibodies in research increases, it is critical that proper antibody authentication experiments are conducted. Funding bodies are also concerned about the apparent lack of experimental reproducibility. In the US, National Institutes of Health requires a validation plan to be provided for key research tools, including antibodies, in all grant applications from 2016 onwards (see http://grants.nih.gov/reproducibility/index.htm).

Collagen VI is present in the extracellular matrix (ECM) of skeletal muscle where it functions to anchor the basement membrane to underlying interstitial tissues. Five collagen VI chains have been described in human $[3,4]$. Mutations in three of these (COL6A1, COL6A2 and COL6A3) result in two congenital muscular 
dystrophies demonstrating that collagen VI is critical for muscle development. These diseases are the relatively mild Bethlem myopathy (BM) and the more severe Ullrich congenital muscular dystrophy (UCMD) $[5,6]$. Studies on skeletal muscle biopsies and cultured fibroblasts isolated from affected individuals have established that, in general, the severity of disease is determined by the type of mutation. The majority of disease-causing mutations are amino acid substitutions that typically result in reduced efficiency of collagen VI assembly, leading to reduced and/or aberrant deposition of collagen VI in the muscle ECM [7-10]. Antibodies are used to assess the consequences of mutant or absent protein on collagen VI assembly by immunoblotting, and on ECM deposition and organization by immunohistochemistry [9, 11-14]. Commonly-used antibodies include VI-26 (MAB3303) raised against tissue-purified collagen VI, 3C4/Mab1944 for the $\alpha 3$ chain, and $6 \mathrm{~A} 1-\mathrm{H} 200$ for $\alpha 1$. Public discussion within the collagen VI community about the use of antibodies for the molecular and histological analysis of collagen VI mutations $[15,16]$ stimulated an effort to authenticate commonly-used antibodies. In this report we define chain specificity and relative sensitivities of 23 commercially-available collagen VI antibodies by immunohistochemistry and immunoblotting.

\section{Methods}

\section{Immunohistochemistry}

Frozen sections of normal human skeletal muscle were obtained from the Michigan Society of Histotechnologists. Tissues were embedded in Tissue-Tek OCT (Sakura) and frozen in isopentane cooled in liquid nitrogen. Tissue blocks were stored at $-80{ }^{\circ} \mathrm{C}$ before sectioning on a Microm HM550 cryostat. Transverse and longitudinal four micron-width sections were mounted on charged slides (Fisher) and stored at $-80{ }^{\circ} \mathrm{C}$ before staining. Serial cryosections were warmed to room temperature and briefly fixed in acetone at $-20{ }^{\circ} \mathrm{C}$ then air-dried and equilibrated in $1 \times$ PBS. Following equilibration, sections were blocked at room temperature with $10 \%(\mathrm{v} / \mathrm{v})$ normal serum of the species the secondary antibody was raised in. Primary antibodies including catalogue number, species of origin, epitope (if provided) and working dilutions for each application are shown in Table 1. Working dilutions were determined via manufacturer recommended dilutions for immunohistochemistry and ranged from 1:40 to 1:400. Fluorescein isothiocyanate (FITC)-conjugated goat anti-mouse IgG (Abcam ab6785) and Cy3-conjugated goat antirabbit IgG diluted (Abcam ab6939) secondary antibodies were used for the majority of the antibody panel. In the case of primary antibody H3-2, a mouse IgM, FITC-conjugated goat anti-mouse IgM diluted (Abcam ab97229) secondary antibody was used. For primary antibodies raised in goat, a Cy2 donkey anti-goat secondary was used (GeneTex GTX26948). Cryosections were incubated with primary and secondary antibodies for $1 \mathrm{~h}$ at room temperature. Sections were washed in $1 \times$ PBS between primary and secondary incubations and stained sections were mounted with ProLong Gold Antifade with DAPI (Cell Signaling Technology) (UV/ nuclear staining not shown). All immunofluorescence images were obtained via Photometrics camera (CoolSNAP MYO) paired with Nikon Eclipse Ti-S fluorescence microscope. Images were analyzed with NIS-Elements software.

\section{Cell transfections}

cDNAs for each chain were PCR amplified from existing cDNA clones and subcloned into a mammalian expression vector (pCDNA6). COL6A1 and COL6A2 were full-length cDNA clones encompassing the $\mathrm{N} 1$ to $\mathrm{C} 2$ domains. The COL6A3 cDNA spanned the N6 to C5 domains and we have previously established that this cDNA assembles into multimeric collagen VI [17]. All clones were verified by Sanger sequencing.

HEK-293 cells (ATCC, CRL-1573) were independently transfected with cDNAs for the human COL6A1, COL6A2 and COL6A3 genes producing four cell lines that stably express the $\alpha 1, \alpha 2$ and $\alpha 3$ chains of collagen VI separately, and one line that expresses all three chains together. We have previously established that HEK293 cells do not express detectable levels of COL6A1, COL6A2 or COL6A3 mRNA by RT-PCR (data not shown).

\section{Immunostaining transfected cells}

HEK-293 cultures expressing each chain individually were grown to confluency in Dulbecco's modified Eagles medium (GIBCO) supplemented with $10 \%$ fetal calf serum (GIBCO) in 4-well chamber slides. Cells were incubated overnight in media supplemented with $0.25 \mathrm{mM}$ sodium ascorbate to facilitate collagen biosynthesis. Cells were fixed in $2 \%$ paraformaldehyde, permeabilized with $0.1 \% \mathrm{v} / \mathrm{v}$ Triton-X100 (Sigma), and blocked with $10 \%$ normal goat serum (Abcam, ab156046) in $1 \times$ PBS. Primary and secondary antibodies were added at the same concentration and incubation times as for the immunohistochemical studies (see Table 1).

\section{Cell lysate preparation}

Transfected HEK-293 cells were grown to confluence in DMEM with $10 \%(\mathrm{v} / \mathrm{v})$ fetal calf serum, then supplemented with $0.25 \mathrm{mM}$ sodium ascorbate for $24 \mathrm{~h}$. Media was removed and the cells gently washed with $1 \times$ PBS to remove residual media. Lysates were prepared by 
Table 1 Summary of collagen VI antibodies and experimental conditions

\begin{tabular}{|c|c|c|c|c|c|c|}
\hline Antibody & Supplier & Cat number & Species & Immunogen & IHC dilution & IB dilution \\
\hline Collagen VI VI-26 & Millipore & MAB3303 & mou mAb & Human-tissue not specified & $1: 100$ & $1: 500$ \\
\hline Collagen VI & Fitzgerald Ind. & 70R-CR009X & rab pAb & Human/bovine placenta & $1: 100$ & $1: 1000$ \\
\hline Collagen VI 172C2 & Santa Cruz & sc-47764 & mou mAb & Human-tissue not specified & $1: 100$ & $1: 500$ \\
\hline Collagen VI 5C6 & U. lowa DSHB & - & mou mAb & Human fetal membranes & $1: 100$ & $1: 500$ \\
\hline Collagen VI & Millipore & AB7821 & rab pAb & Human placenta & $1: 100$ & $1: 500$ \\
\hline COL6A1 H200 & Santa Cruz & sc-20649 & rab pAb & a1 chain, amino acid 51-250 & $1: 40$ & $1: 500$ \\
\hline COL6A1 & Abcam & ab199720 & $\mathrm{rab} \mathrm{mAb}$ & a1 chain, within aa 800 to C-term. & $1: 400$ & $1: 2000$ \\
\hline COL6A1 & Abcam & ab182744 & $\mathrm{rab} \mathrm{mAb}$ & a1 chain within amino acid 1-250 & $1: 100$ & $1: 1000$ \\
\hline COL6A1 & Proteintech Gp & 17023-1-AP & rab pAb & Not available & $1: 100$ & $1: 500$ \\
\hline COL6A1 B4 & Santa Cruz & sc-377143 & mou mAb & a1 chain amino acid 53-86 & $1: 40$ & $1: 500$ \\
\hline COL6A2 & Proteintech Gp & 14853-1-AP & $\mathrm{rab} \mathrm{pAb}$ & Not available & $1: 100$ & $1: 500$ \\
\hline COL6A2 EPR7889 & Abcam & ab180855 & $\mathrm{rab} \mathrm{mAb}$ & a2 chain, amino acid 800-900 & $1: 400$ & $1: 1000$ \\
\hline COL6A2 & Abcam & $a b 172606$ & $\mathrm{rab} \mathrm{mAb}$ & a2 chain, amino acid 200-300 & $1: 100$ & $1: 1000$ \\
\hline COL6A2 D20 & Santa Cruz & sc-83607 & $\mathrm{rab} \mathrm{pAb}$ & a2 chain, internal epitope & $1: 40$ & $1: 500$ \\
\hline COL6A2 H300 & Santa Cruz & sc-292186 & rab pAb & a2 chain, amino acid $241-540$ & $1: 40$ & $1: 500$ \\
\hline COL6A2 K15 & Santa Cruz & sc-377143 & $\mathrm{rab} \mathrm{pAb}$ & a2 chain, C-terminus & $1: 40$ & $1: 500$ \\
\hline COL6A2 B7 & Santa Cruz & sc-374566 & mou mAb & a2 chain, amino acid 241-540 & $1: 40$ & $1: 500$ \\
\hline COL6A3 G18 & Santa Cruz & sc-131139 & goat pAb & Not available & $1: 40$ & $1: 500$ \\
\hline COL6A3 3C4 & Millipore & MAB1944 & mou mAb & Not available & $1: 100$ & $1: 1000$ \\
\hline COL6A3 3C4 & Santa Cruz & sc-47712 & mou mAb & Not available & $1: 100$ & $1: 250$ \\
\hline COL6A3 H300 & Santa Cruz & sc-367543 & rab pAb & a3 chain, amino acid 301-600 & $1: 40$ & $1: 500$ \\
\hline COL6A3 H3-2 & Santa Cruz & sc-81766 & mou mAb & a3 chain, globular domains & $1: 100$ & $1: 500$ \\
\hline COL6A3 64C11 & Abcam & ab49273 & mou mAb & a3 chain, 295 aa C-term. frag & $1: 100$ & $1: 1000$ \\
\hline COL6A3 N12 & Santa Cruz & sc-131140 & goat pAb & Not available & $1: 40$ & $1: 500$ \\
\hline
\end{tabular}

Antibodies are grouped by name and clone and each entry includes supplier and catalogue number, species antibody was raised in and whether the antibody is monoclonal or polyclonal. Also listed is the immunogen/epitope used to generate the antibody, if known, and the dilutions used for each application

scraping the adherent cell layer into $1 \%$ Nonidet-P40 (Sigma) in $1 \times$ PBS with EDTA-free protease inhibitor cocktail (Roche). Lysates were incubated under agitation for $24 \mathrm{~h}$ at $4{ }^{\circ} \mathrm{C}$, centrifuged to pellet insoluble material, and the supernatants collected. To ensure equal gel loading, sample protein concentration were determined using a BCA assay kit (Thermo Scientific) and aliquots were resolved under reducing (20 mM DTT) and non-reducing conditions by SDS-PAGE. Gels were either $6 \%(\mathrm{w} / \mathrm{v})$ polyacrylamide made in-house or $4-15 \%(\mathrm{w} / \mathrm{v})$ polyacrylamide gradient gels purchased from Bio-Rad.

\section{Immunoblotting}

For immunoblotting, collagen chains resolved by SDSPAGE were transferred onto PVDF membranes (Millipore), then blocked for $1 \mathrm{~h}$ at room-temperature in $4 \%$ nonfat dried milk in $0.1 \%$ PBS/tween-20. Blots were probed individually with collagen VI antisera, some chain-specific and some raised against tissue-purified collagen VI. Antibody dilutions are listed in Table 1. Bound primary antibody was detected with either antigoat, -rabbit, or-mouse fluorescently-labeled secondary
IgG. Blots were visualized on the Odyssey CLx imager (LiCor).

All immunoblot and immunohistochemistry experiments were repeated at least once to confirm results.

\section{Results}

To assess the relative efficiency of collagen VI antibodies to detect collagen VI in muscle, cross and longitudinal frozen sections of normal human skeletal muscle were immunostained with a panel of 23 commerciallyavailable collagen VI antibodies. Primary antibodies were detected by fluorescently-labelled secondary antibodies. Experimental conditions were identical except for antibody dilutions, which were selected based on manufacturers guidelines, and choice of secondary antibody which was dictated by the species the primary antibody was raised in.

To validate the collagen VI antibodies for use in immunoblot studies, we developed four HEK-293 fibroblast cell lines; three that express $\alpha 1, \alpha 2$ and $\alpha 3$ individually and one that expresses all three together. The commonly accepted dogma is that in cells that endogenously 
produce collagen VI, the three chains co-assemble into heterotrimers in a 1:1:1 stoichiometric ratio $[18,19]$. We assume that the collagen VI produced by the tripletransfected cells assemble in accordance with this dogma, although other assemblies may be possible. Using this panel it is possible to determine, (1) whether the antibodies recognize the correct individual collagen VI chains and/or multimeric collagen VI, (2) whether they cross-react with other closely-related collagen VI chains and (3), their relative sensitivities in detecting the same $\alpha$ chain. A summary of results from the immunohistochemical and immunoblot experiments is presented in Table 2.

Chain specificity was also confirmed for three antibodies by immunocytochemistry on transfected cells (Additional file 1: Figure S1). These antibodies were; ab199720 for $\alpha 1$, ab180855 for $\alpha 2$ and 70R-CR009X for $\alpha 3$. While these cells do not produce a collagen VI extracellular matrix because they lack the other chains necessary for heterotrimeric assembly, they are used in the current study to confirm chain specificity as indicated by immunoblot data.

\section{Antibodies raised against tissue-purified collagen VI}

Five antibodies that were raised against collagen VI from pepsin-treated tissue were tested (Fig. 1). Of these, only two showed detectable signals by immunohistochemistry: monoclonal MAB3303 (clone VI-26 from Millipore) (panels A and B) and polyclonal 70R-CR009X (Fitzgerald Industries) (E and F). The staining was clearly pericellular in cross- and longitudinal sections, as expected for collagen VI. In addition, both antibodies recognized high molecular weight collagen VI in lysates from cells transfected with all three chains (D and $\mathrm{H})$. Interestingly, the Fitzgerald Industries antibody also recognized individual $\alpha 3$ chains when electrophoresed under nonreducing conditions suggesting that the $\alpha 3$ chain may be the major epitope for this antibody $(\mathrm{G})$. This finding was confirmed in transfected cells where 70R-CR009X stained cells expressing $\alpha 3$ chains but not those expressing $\alpha 1$ nor $\alpha 2$ chains (Additional file 1: Figure S1, panels $\mathrm{C}, \mathrm{H}$ and $\mathrm{M}$ ). This is surprising since native collagen VI was used as the immunogen and may suggest that $\alpha 3$ chains are 'preferentially' exposed on native collagen VI fibrils. Alternatively, the $\alpha 3$ chain may have a more

Table 2 Summary of immunohistochemistry and immunoblot results

\begin{tabular}{|c|c|c|c|c|c|c|c|}
\hline Antibody & Cat number & Signal by IHC & a1 lysate & a2 lysate & a3 lysate & a1 a2 a3 lysate & Figures \\
\hline COL6A1 H200 & sc-20649 & ++ & $++(\mathrm{R})$ & - & - & + & 1 \\
\hline COL6A1 & ab199720 & +++ & $+++(R) /+++(N R)$ & - & - & + & 1 \\
\hline COL6A1 & ab182744 & ++ & $++(\mathrm{R})$ & - & - & + & 1 \\
\hline COL6A1 & 17023-1-AP & +++ & $+++(\mathrm{R}) /+++(\mathrm{NR})$ & - & - & + & 1 \\
\hline COL6A1 B4 & sc-377143 & - & $+++(\mathrm{R})$ & - & - & + & 1 \\
\hline COL6A2 & 14853-1-AP & ++ & - & $++(\mathrm{R}) /+(\mathrm{NR})$ & - & nd & 2 \\
\hline COL6A2 EPR7889 & ab180855 & +++ & - & $+++(R)$ & - & - & 2 \\
\hline COL6A2 & ab172606 & ++ & - & $+(R)$ & - & nd & 2 \\
\hline COL6A2 D20 & sc-83607 & +++ & - & $+++(R)$ & - & ++ & 2 \\
\hline COL6A2 H300 & sc-292186 & ++ & - & $+(\mathrm{R})$ & - & + & 2 \\
\hline COL6A2 K15 & sc- 377143 & +++ & - & $+(\mathrm{R})$ & - & + & 2 \\
\hline COL6A2 B7 & sc- 374566 & - & - & $+++(\mathrm{R}) /+(\mathrm{NR})$ & - & ++ & 2 \\
\hline COL6A3 G18 & sc-131139 & ++ & - & - & $+(\mathrm{NR})$ & + & 3 \\
\hline COL6A3 3C4 & MAB1944 & +++ & - & - & $+(\mathrm{R}) /++(\mathrm{NR})$ & - & 3 \\
\hline COL6A3 3C4 & sc- -47712 & & - & - & + & nd & 3 \\
\hline COL6A3 H300 & sc-367543 & +++ & - & - & - & - & 3 \\
\hline COL6A3 H3-2 & sc- 81766 & - & - & - & - & - & 3 \\
\hline COL6A3 64C11 & ab49273 & - & - & - & - & - & 3 \\
\hline COL6A3 N12 & sc-131140 & - & - & - & - & nd & 3 \\
\hline Collagen VI VI-26 & MAB3303 & +++ & - & - & - & + & 4 \\
\hline Collagen VI & 70R-CR009X & +++ & - & - & $++(\mathrm{NR})$ & +++ & 4 \\
\hline Collagen VI 172C2 & sc-47764 & - & - & - & $++(N R)$ & - & 4 \\
\hline Collagen VI 5C6 & & - & - & - & - & + & 4 \\
\hline Collagen Vl & AB7821 & - & - & Non-sp. & Non-sp. & ++ & 4 \\
\hline
\end{tabular}

Each antibody is scored for signal intensity on immunohistochemical signal and, for immunoblots, band intensity ranging from '-'representing no detectable signal, to' +++ ' representing strong signal. The description of immunoblots signal strength scores reduced (R) and non-reduced (NR) samples separately. Some blots on triple transfected lysates were not conducted and are designated as not determined (nd) 


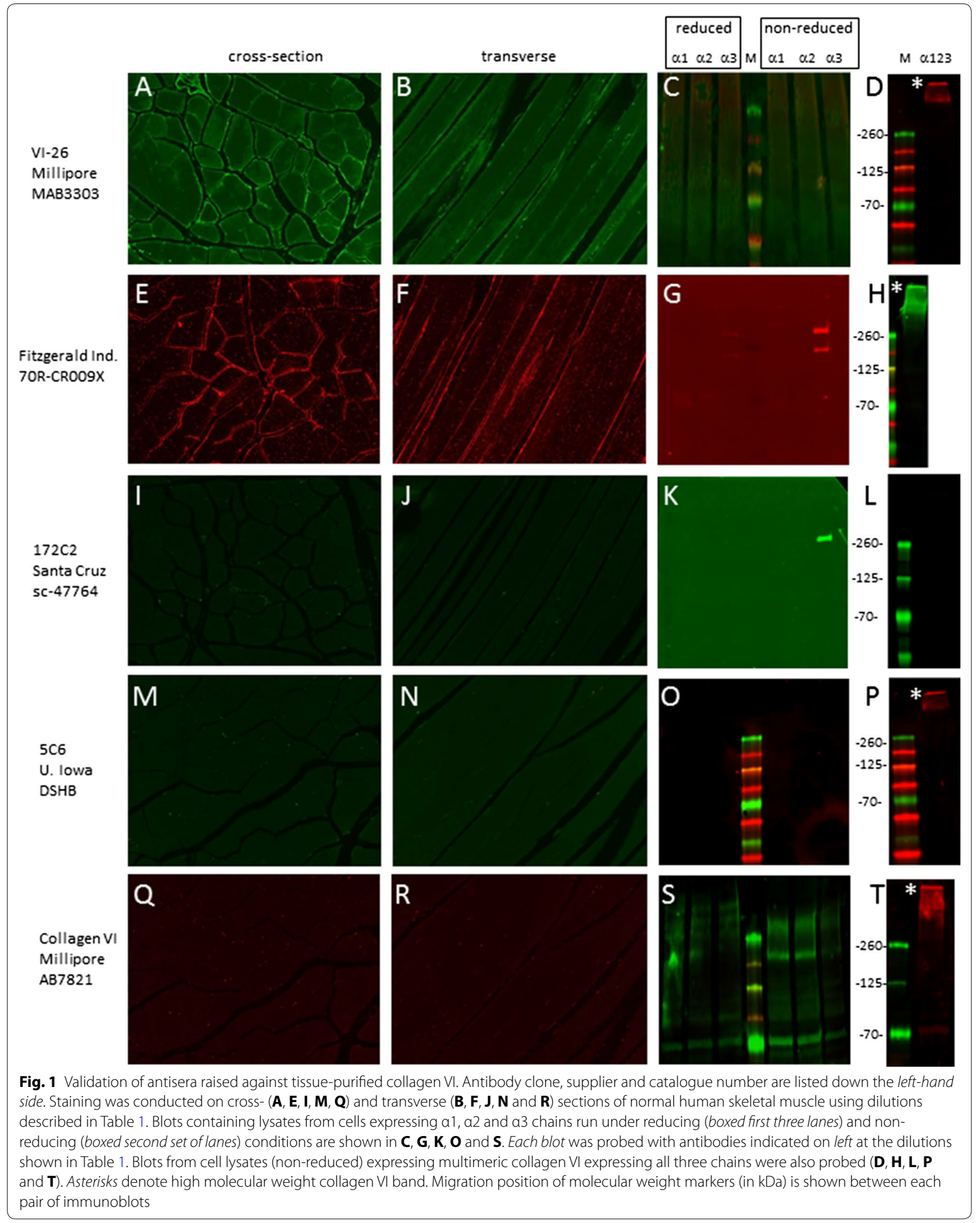


immunogenic structure than $\alpha 1$ and $\alpha 2$ chains in native collagen VI. Of the antibodies that failed to stain tissue, 5 C6 is of interest because it has been used previously in immuno-gold labeling experiments to detect collagen VI in skin and cartilage $[20,21]$ where presumably collagen VI structures are in their native form. It is unclear why $5 \mathrm{C6}$ failed to stain muscle frozen tissue ( $\mathrm{M}$ and $\mathrm{N}$ ) but the $5 \mathrm{C} 6$ epitope may not be revealed by the fixation and section preparation used in this study, or alternatively, skin and cartilage collagen VI has a different supramolecular architecture than muscle collagen VI and epitope accessibility varies between the two tissues. The sc- 47764 antibody (clone $172 \mathrm{C} 2$ from Santa Cruz) did not detect muscle collagen VI by immunohistochemistry (I and J) but recognized individual $\alpha 3$ chains by blotting (K). The Millipore antibody AB7821 did not detect collagen VI by immunohistochemistry and reacted with multiple bands by immunoblotting $(\mathrm{Q}-\mathrm{T})$.

\section{Antibodies directed against individual collagen VI chains a1(VI)}

Four of the five antibodies against the $\alpha 1$ chain produced a signal on skeletal muscle sections, with ab199720 (abcam) (Fig. 2, panels E, F) and 17023-1-AP (ProteinTech Group) ( $\mathrm{M}$ and $\mathrm{N}$ ) detecting highest levels in the pericellular space. The sc-20649 (clone H200 from Santa Cruz) staining intensity was weaker and intracellular structures appeared to be positive (A and B). Chain specificity immunoblots revealed that all five anti- $\alpha 1$ antibodies tested recognized $\alpha 1$ chains, and no other chains, when resolved under reducing conditions at the predicted molecular weight of $140 \mathrm{kDa}(\mathrm{C}, \mathrm{G}, \mathrm{K}, \mathrm{O}$ and S). Antibodies 17023-1-AP and ab199720 recognized $\alpha 1$ chains in both reduced and non-reduced samples (G and $\mathrm{O}$ ). Interestingly, both these antibodies revealed the presence of discrete $\alpha 1$ oligomers larger than $140 \mathrm{kDa}$ suggesting that $\alpha 1$-chains may undergo self-assembly to form homo-oligomers in these cells. Four antibodies recognized $\alpha 1$ chains at approximately $180 \mathrm{kDa}$ in lysate containing all three chains which corresponds to the size of $\alpha 1$ dimers (D, H, L and P). All $\alpha 1$ antibodies recognize a small amount of high-molecular weight collagen VI near the top of the blots.

In transfected cells, ab199720 gave a strong signal in cultures of cells expressing $\alpha 1$, but not in cells expressing $\alpha 2$ or $\alpha 3$ chains (Additional file 1: Figure S1, panels $\mathrm{A}, \mathrm{F}$ and $\mathrm{K}$ ). This confirms the immunoblot finding (see Fig. 2G) that this antibody specifically recognizes the $\alpha 1$ chain.

\section{a2(VI)}

Six out of seven $\alpha 2$-antisera stained skeletal muscle, with ab180855 (abcam) demonstrating the strongest signal
(Fig. 3, panel D). Only sc-374566 (clone B7 from Santa Cruz) failed to detect muscle collagen VI (W and X). All seven $\alpha 2$ antibodies recognized their epitopes when lysates were resolved under reducing conditions although with a range of sensitivities. Three $\alpha 2$ antisera worked well (F, M and Y) with clearly detectable bands at the correct molecular weight $(140 \mathrm{kDa})$ and four performed poorly with only faint $\alpha 2$ bands present (C, J, Q and $U$ ). Only sc-374566 recognized individual non-reduced $\alpha 2$ chains, albeit weakly $(\mathrm{Y})$. Several antibodies recognized multimeric collagen VI with sc-374566, sc-83607 (clone D20 from Santa Cruz), and sc-377143 (clone K15 from Santa Cruz) (asterisks in panels N, V and Z) showing the strongest signals. ab180855 stained cells expressing $\alpha 2$ but not $\alpha 1$ nor $\alpha 3$ (Additional file 1: Figure S1, panels B, $G$ and $L$ ) confirming immunoblot findings for this antibody (Fig. 3F).

\section{a3(VI)}

Three of six $\alpha 3$ antibodies stained skeletal muscle including the commonly used 3C4/MAB1944 (Millipore) which also appeared to stain intracellular structures (Fig. 4 panels A, B, E, F, I and J). 3C4/MAB1944 and sc-131139 (clone G18) recognized their $260 \mathrm{kDa}$ target under nonreducing conditions [22, 23] ( $\mathrm{C}$ and $\mathrm{G}$ ). The remaining $\alpha 3$ antibodies failed to recognize trimeric collagen VI nor individual chains under reducing or non-reducing conditions. Three antisera; sc-81766 (clone H3-2 from Santa Cruz), ab49273 (clone 64C11 from Abcam) and sc-131140 (clone N12 from Santa Cruz) failed to recognize collagen VI by either method using the experimental conditions used in this study ( $\mathrm{M}$ to $\mathrm{V}$ ).

In the course of these studies we became aware that the mouse monoclonal antibody 3C4/MAB1944, which recognizes the $\alpha 3(\mathrm{VI})$ chain, gave different results depending on supplier and batch number (Fig. 5). As shown in Fig. $5 \mathrm{C}, 3 \mathrm{C} 4$ from Santa Cruz recognized $\alpha 3(\mathrm{VI})$ at the correct molecular weight of $260 \mathrm{kDa}$ when resolved under reducing conditions. In contrast, the same antibody clone from Millipore recognized $\alpha 3$ at $260 \mathrm{kDa}$ and additional bands at 50, 70 and $90 \mathrm{kDa}$ depending on lot number (Fig. 5A, B). 3C4 is widely used in studies on human muscle using immunohistochemistry. Our data suggest that care should be taken in interpreting data using these batches of antibody from Millipore.

\section{Controls}

Control experiments where sections were stained using rabbit, mouse and goat IgGs at dilutions of 1:100 did not detect any collagen VI (Additional file 2: Figure S2, panels A-F). Similarly, blots containing lysates from triple transfected cells probed with mouse IgG and normal rabbit and goat serum failed to detect significant bands $(\mathrm{G}-\mathrm{I})$. 


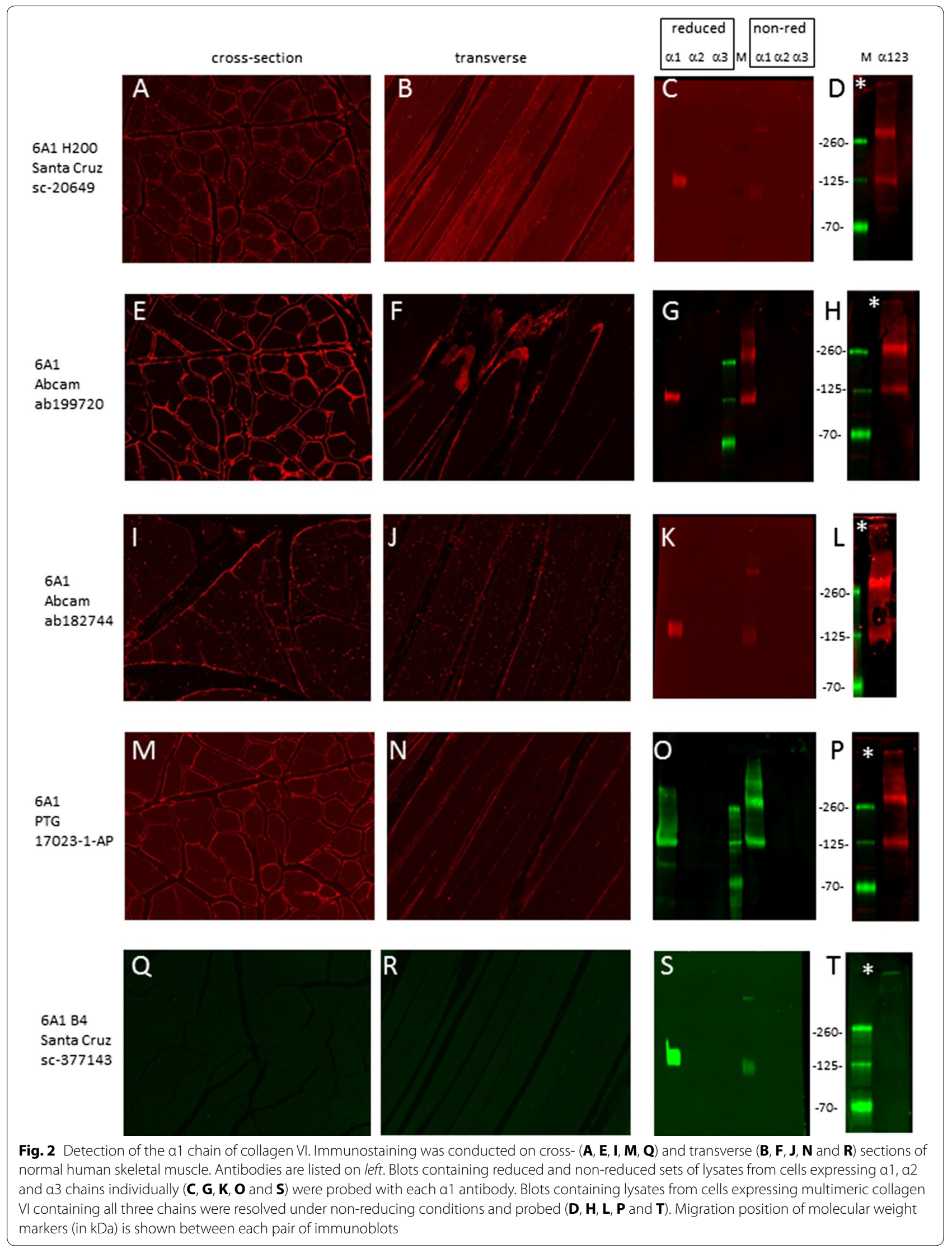




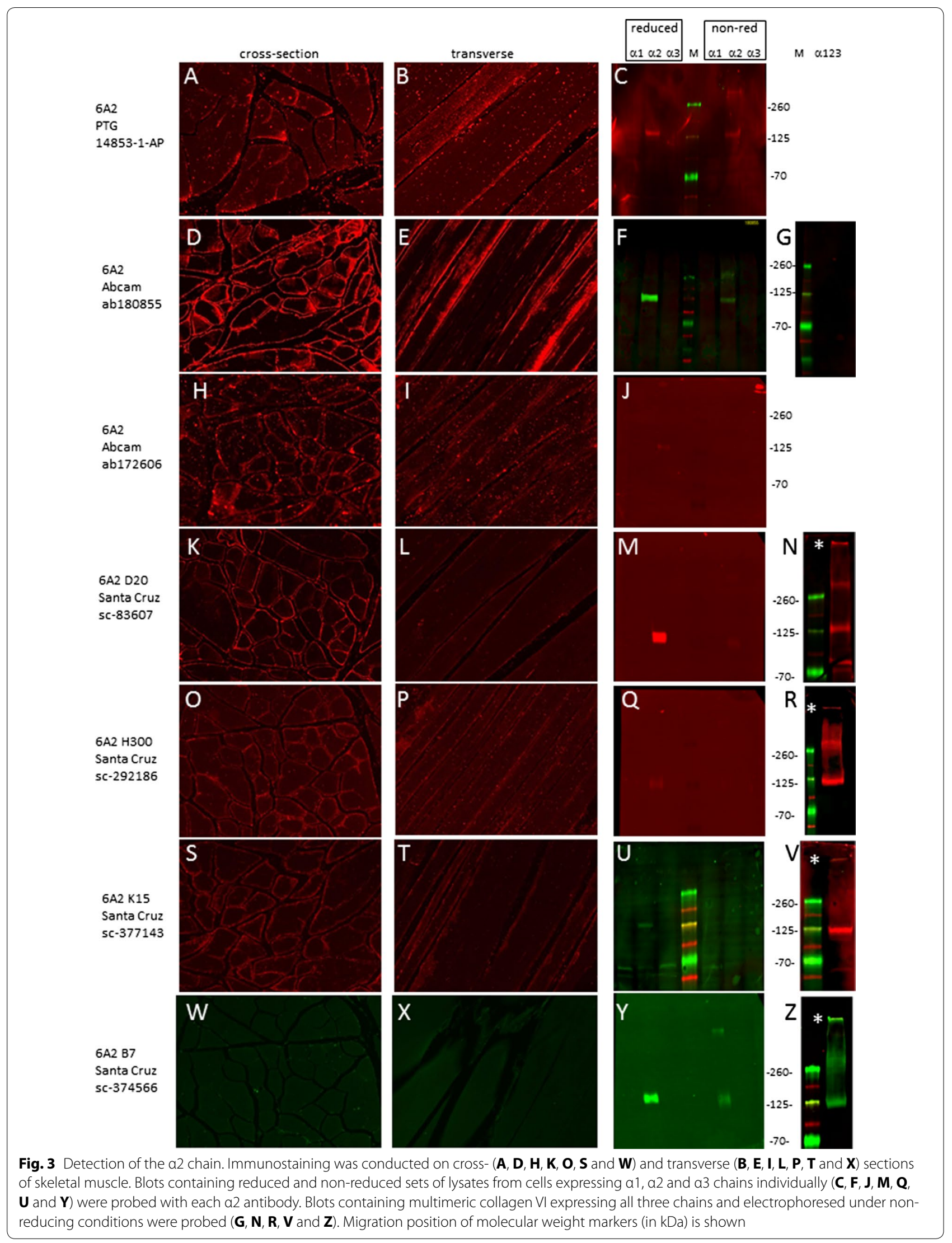




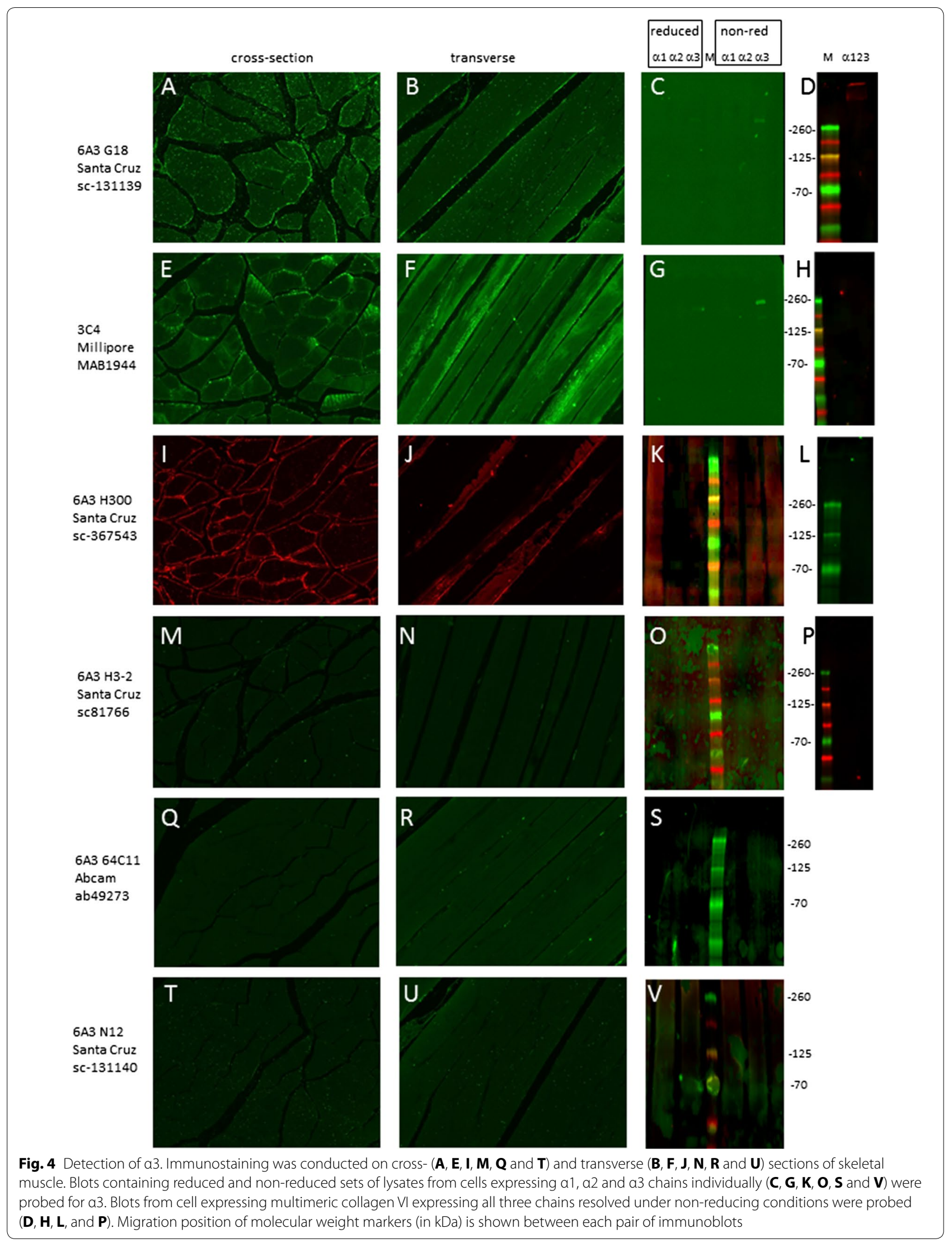




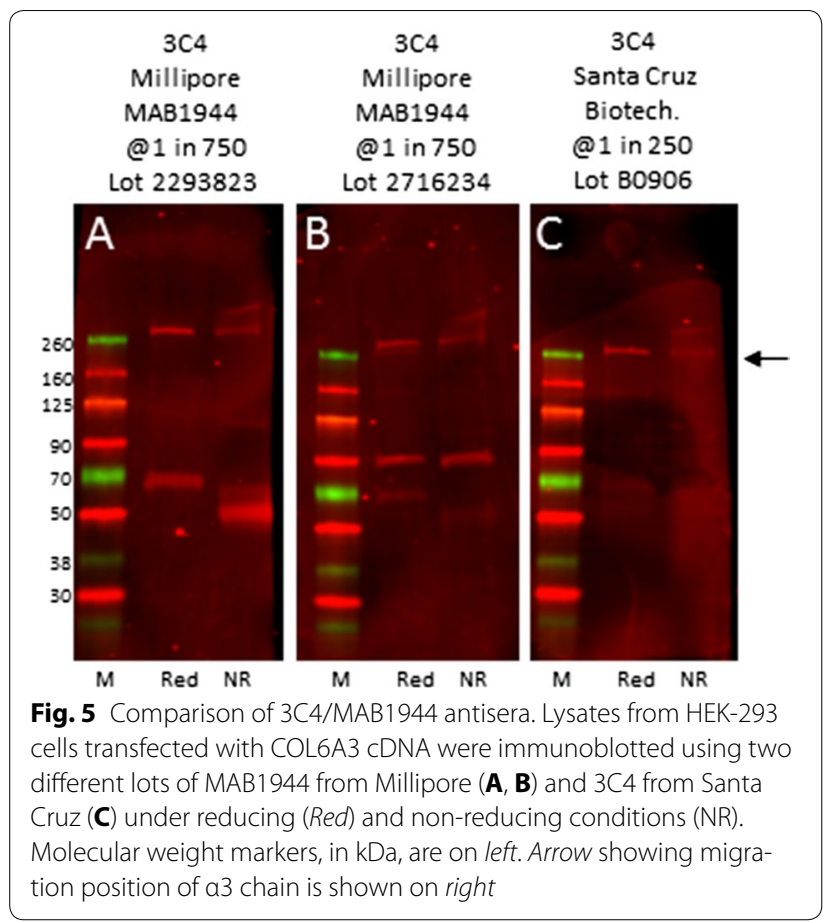

A faint band at $70 \mathrm{kDa}$ is present on the blot probed with normal rabbit serum but this is smaller than the $140 \mathrm{kDa}$ $\alpha 1$ and $\alpha 2$ bands and $260 \mathrm{kDa} \alpha 3$ bands and represents background (asterisk in panel $\mathrm{H}$ ).

\section{Discussion}

To provide a meaningful assessment of the relative usefulness of collagen VI antibodies for immunohistochemistry 23 collagen VI antibodies from Abcam, Santa Cruz, ProteinTech Group and Millipore were tested for reactivity on human muscle sections under standard conditions. Since aberrant collagen VI tissue deposition can be caused by mutations in three genetically-distinct collagen VI chains and it would be valuable to know whether any antibody cross-reacted with the other structurally-similar chains, each antibody was tested for chain specificity by immunoblot analyses and, for three antibodies, immunocytochemistry. Comparative testing revealed that 15 out of 23 antibodies detected pericellular collagen VI on human skeletal muscle sections. The best antibodies specific for each chain are: ab199720 and 17023-1-AP for $\alpha 1$, ab180855 and 14853-1-AP for $\alpha 2,3 C 4 / M A B 1944$ and sc-367543 for $\alpha 3$ (see Table 2). The only two antibodies raised against tissue collagen VI that detected multimeric collagen VI by immunocytochemistry were MAB3033 and 70R-CR009x.

14 out of 18 chain-specific antibodies recognized their target collagen VI chains although with a wide range of sensitivities. Notably, all 14 antibodies demonstrated chain specificity with each antisera recognizing a single collagen VI chain and none appeared to cross-react with the other collagen VI chains. However, good specificity within the collagen VI family does not exclude the possibility that these antibodies bind to other collagens or non-collagenous proteins. Each distinct chain was recognized by at least two different antibodies with high sensitivity (Table 2). The finding that antisera against $\alpha 1$ and $\alpha 2$ primarily recognizes the reduced form of the chain suggests that these chains form intra- or inter-chain disulfide bonds that nominally mask the epitopes, and complete reduction of disulfides is essential for optimal antibody recognition. Epitopes within high molecular weight collagen VI were detected by multiple antibodies with the three strongest signals being ab182744 against $\alpha 1$, and sc- 374566 and sc- 83607 against $\alpha 2$.

There has been discussion in the collagen VI literature regarding the use of VI-26/MAB3303 and 3C4 for immunostaining patient muscle tissue and fibroblasts $[15,16]$. It has been reported that VI-26 is less sensitive at detecting collagen VI in muscle and fibroblast preparations compared to 3C4 leading to an underestimation of the amount of collagen VI degradation [23]. Our data indicates that they recognize different forms of collagen VI; VI-26 detects high-molecular weight, (presumably) triple helical collagen VI but not any individual chains (Fig. 1C, D) whereas $3 \mathrm{C} 4$ detects the $\alpha 3$ chain and not collagen VI multimers (Fig. 4G, H). These antibodies clearly recognize different epitopes and may detect different subpopulations of collagen VI fibrils in the ECM. We suggest staining using more than one antibody when detecting collagen VI by immunohistochemistry.

\section{Conclusions}

This study expands the list of collagen VI antibodies that can be used in immunoblotting for the analysis of collagen VI on patient samples. Antibodies with high specificity and sensitivity are available for each collagen VI chain. Most $\alpha 1$ and $\alpha 2$ antisera perform best on reduced material; in contrast the majority of $\alpha 3$ antisera tested performed better on non-reduced samples. Several antibodies recognize multimeric collagen VI with the strongest signal from the 70R-CR009X antibody.

There have been calls to standardize antibody production, characterization and use [24, 25]. It is recommended that journals insist that authors be more transparent and consistent with reporting antibody details [26]. We echo this sentiment and reiterate the need to include basic information when reporting antibody experiments by including dilutions used, vendor and catalogue/clone numbers, and species antibody was raised in. In addition, it is essential to include controls such as isotype controls for monoclonal antibodies, pre-immune serum controls 
for polyclonal antisera and blocking peptide controls for peptide antibodies, if possible.

\section{Additional files}

Additional file 1: Figure S1. Immunostaining transfected cells for antibody specificity. HEK-293 cells transfected with cDNAs for COL6A1 (panels A-E), COL6A2 (F-J) and COL6A3 (K-O) were stained for a1 chains ( $A$, F and $\mathrm{K})$, a2 chains ( $\mathrm{B}, \mathrm{G}$ and $\mathrm{L})$ and collagen $\mathrm{VI}(\mathrm{C}, \mathrm{H}$ and $\mathrm{M})$. Negative control stains for rabbit $(D, I$ and $N)$ and mouse $(E, J$ and $O)$ lgGs are shown. Each antibody recognizes its correct chain and not the other chains.

Additional file 2: Figure S2. Experimental controls for immunohistochemical and immunoblot experiments. Normal human skeletal muscle cross- and transverse sections were stained for rabbit (panels A and B), mouse ( $C$ and $D$ ) and goat ( $E$ and $F$ ) IgGs were used at a dilution of 1 in 100 and detected using the indicated fluorescently-labelled secondary antibodies. All controls were blank. Blots containing lysates from HEK-293 cells transfected with all three chains were probed for mouse IgG (panel $\mathrm{G}$ ), normal rabbit (H) or normal goat (I) serum. Migration positions of molecular weight markers are shown on left. A faint band at $70-75 \mathrm{kDa}$ is present in panel $\mathrm{H}$ (indicated by asterisk).

\section{Authors' contributions}

JE conducted the immunohistochemical, immunocytochemical and the immunoblotting analyses. PH conducted the immunoblotting analyses. JF wrote the manuscript with input from JE and PH. All authors read and approved the final manuscript.

\section{Author details}

1 Bone and Joint Center, Department of Orthopedic Surgery, Henry Ford Hospital System, Integrative Biosciences Building, 6135 Woodward Ave, Detroit, MI 48202, USA. ${ }^{2}$ Department of Orthopaedics and Rehabilitation, Oregon Health and Science University, Portland, OR 97239, USA.

\section{Acknowledgements}

The authors would like to thank LaToya Jackson for providing paraffin sections.

\section{Competing interests}

The authors declare that they have no competing interests.

\section{Availability of data and materials}

All data generated or analyzed during this study are included in this published article and its additional information files. All antibodies are commercially available.

\section{Consent for publication}

Informed consent was obtained from all tissue donors.

\section{Ethics approval and consent to participate}

Research on human material was conducted in accordance with the Declaration of Helsinki. Human tissue was collected from the Michigan Society of Histotechnologists who have the authority to supply researchers with human material.

\section{Funding}

This work was supported by the National Institute of Arthritis and Musculoskeletal and Skin Diseases of the National Institutes of Health under Award Number R01AR055957 (to J.F.).

\section{Publisher's Note}

Springer Nature remains neutral with regard to jurisdictional claims in published maps and institutional affiliations.

Received: 19 October 2016 Accepted: 22 July 2017

Published online: 29 July 2017
References

1. Begley CG, Ellis LM. Drug development: raise standards for preclinical cancer research. Nature. 2012;483:531-3.

2. Berglund L, Bjorling E, Oksvold P, Fagerberg L, Asplund A, Szigyarto CA, Persson A, Ottosson J, Wernerus H, Nilsson P, et al. A genecentric Human Protein Atlas for expression profiles based on antibodies. Mol Cell Proteom. 2008;7:2019-27.

3. Fitzgerald J, Rich C, Zhou FH, Hansen U. Three novel collagen VI chains, alpha4(VI), alpha5(VI), and alpha6(VI). J Biol Chem. 2008;283:20170-80.

4. Gara SK, Grumati P, Urciuolo A, Bonaldo P, Kobbe B, Koch M, Paulsson M, Wagener R. Three novel collagen VI chains with high homology to the alpha3 chain. J Biol Chem. 2008;283:10658-70.

5. Bonnemann CG. The collagen VI-related myopathies: muscle meets its matrix. Nat Rev Neurol. 2011;7:379-90.

6. Lampe AK, Bushby KM. Collagen VI related muscle disorders. J Med Genet. 2005;42:673-85.

7. Butterfield RJ, Foley AR, Dastgir J, Asman S, Dunn DM, Zou Y, Hu Y, Donkervoort S, Flanigan KM, Swoboda KJ, et al. Position of glycine substitutions in the triple helix of COL6A1, COL6A2, and COL6A3 is correlated with severity and mode of inheritance in collagen VI myopathies. Hum Mutat. 2013;34:1558-67.

8. Lamande SR, Morgelin M, Selan C, Jobsis GJ, Baas F, Bateman JF. Kinked collagen VI tetramers and reduced microfibril formation as a result of Bethlem myopathy and introduced triple helical glycine mutations. J Biol Chem. 2002;277:1949-56.

9. Baker NL, Morgelin M, Peat R, Goemans N, North KN, Bateman $J F$, Lamande SR. Dominant collagen VI mutations are a common cause of Ullrich congenital muscular dystrophy. Hum Mol Genet. 2005;14:279-93.

10. Baker NL, Morgelin M, Pace RA, Peat RA, Adams NE, Gardner RJ, Rowland LP, Miller G, De Jonghe P, Ceulemans B, et al. Molecular consequences of dominant Bethlem myopathy collagen VI mutations. Ann Neurol. 2007:62:390-405.

11. Zamurs LK, Idoate MA, Hanssen E, Gomez-lbanez A, Pastor P, Lamande SR. Aberrant mitochondria in a Bethlem myopathy patient with a homozygous amino acid substitution that destabilizes the collagen VI alpha2(VI) chain. J Biol Chem. 2015;290:4272-81.

12. Lamande SR, Bateman JF, Hutchison W, McKinlay Gardner RJ, Bower SP, Byrne E, Dahl HH. Reduced collagen VI causes Bethlem myopathy: a heterozygous COL6A1 nonsense mutation results in mRNA decay and functional haploinsufficiency. Hum Mol Genet. 1998;7:981-9.

13. Lampe AK, Zou Y, Sudano D, O'Brien KK, Hicks D, Laval SH, Charlton R, Jimenez-Mallebrera C, Zhang RZ, Finkel RS, et al. Exon skipping mutations in collagen $\mathrm{VI}$ are common and are predictive for severity and inheritance. Hum Mutat. 2008;29:809-22.

14. Pan TC, Zhang RZ, Sudano DG, Marie SK, Bonnemann CG, Chu ML. New molecular mechanism for Ullrich congenital muscular dystrophy: a heterozygous in-frame deletion in the COL6A1 gene causes a severe phenotype. Am J Hum Genet. 2003;73:355-69.

15. Sabatelli P, Gualandi F, Bonaldo P, Merlini L. Detecting collagen VI in Bethlem myopathy. J Biol Chem. 2015;290:8011.

16. Lamande SR. Reply to Sabatelli et al.: detecting collagen VI in Bethlem myopathy. J Biol Chem. 2015;290:8012.

17. Fitzgerald J, Morgelin M, Selan C, Wiberg C, Keene DR, Lamande $\mathrm{SR}$, Bateman JF. The N-terminal N5 subdomain of the alpha 3(VI) chain is important for collagen VI microfibril formation. J Biol Chem. 2001;276:187-93.

18. Furthmayr H, Wiedemann $H$, Timpl R, Odermatt E, Engel J. Electron-microscopical approach to a structural model of intima collagen. BiochemJ. 1983;211:303-11.

19. Engel J, Furthmayr H, Odermatt E, von der Mark H, Aumailley M, Fleischmajer R, Timpl R. Structure and macromolecular organization of type VI collagen. Ann NY Acad Sci. 1985;460:25-37.

20. Keene DR, Engvall E, Glanville RW. Ultrastructure of type VI collagen in human skin and cartilage suggests an anchoring function for this filamentous network. J Cell Biol. 1988;107:1995-2006.

21. Hessle H, Engvall E: Type VI collagen. Studies on its localization, structure, and biosynthetic form with monoclonal antibodies. JBiolChem 1984, 259:3955-3961. 
22. Merlini L, Martoni E, Grumati P, Sabatelli P, Squarzoni S, Urciuolo A, Ferlini A, Gualandi F, Bonaldo P. Autosomal recessive myosclerosis myopathy is a collagen VI disorder. Neurology. 2008;71:1245-53.

23. Jimenez-Mallebrera C, Maioli MA, Kim J, Brown SC, Feng L, Lampe AK, Bushby K, Hicks D, Flanigan KM, Bonnemann C, et al. A comparative analysis of collagen $\mathrm{VI}$ production in muscle, skin and fibroblasts from 14 Ullrich congenital muscular dystrophy patients with dominant and recessive COL6A mutations. Neuromuscul Disord. 2006;16:571-82.
24. Bradbury A, Pluckthun A. Reproducibility: standardize antibodies used in research. Nature. 2015;518:27-9.

25. Helsby MA, Fenn JR, Chalmers AD. Reporting research antibody use: how to increase experimental reproducibility. F1000Res. 2013;2:153.

26. Gore AC. Editorial: antibody validation requirements for articles published in endocrinology. Endocrinology. 2013;154:579-80.

\section{Submit your next manuscript to BioMed Central and we will help you at every step:}

- We accept pre-submission inquiries

- Our selector tool helps you to find the most relevant journal

- We provide round the clock customer support

- Convenient online submission

- Thorough peer review

- Inclusion in PubMed and all major indexing services

- Maximum visibility for your research

Submit your manuscript at

www.biomedcentral com/submit 